Received: 2018-05-13

Accepted: 2020-01-21

Published: 2020-07-30

\section{Spinal Cord Stimulation in the Treatment of Neuropathic Pain Associated with Syrinx Developed after Cervical Epidural Steroid INJECTION}

\author{
Anastasios Sakellariou, MD, DMD ${ }^{1}$ \\ Lucien Alexander MD, PhD² \\ Jatinder S. Gill, MD ${ }^{1,3}$ \\ Thomas T. Simopoulos, MD ${ }^{1,3}$
}

Background: We present a case demonstrating the utility of spinal cord stimulation (SCS) in the treatment of neuropathically mediated pain due to cervical spinal cord syringomyelia formation after inadvertent spinal cord injection during interlaminar cervical epidural steroid injection (ICESI). A review of the current literature on the use of cervical SCS for neuropathic pain that is nonradicular is summarized.

Case Report: A 52-year-old woman had a series of ICESIs for cervicalgia complicated by an intraparenchymal injection that resulted in syrinx formation. She subsequently developed severe, permanent right upper extremity spasticity and chronic neuropathic pain. After failure of various conservative, interventional, and surgical treatments, an SCS trial was performed.

Conclusions: SCS may be a viable option for the treatment of neuropathic pain secondary to syringomyelia due to iatrogenic parenchymal injury.

Key words: Neuropathic pain, spinal cord stimulation, syringomyelia following trauma, syrinx

From: 'Beth Israel Deaconess Medical Center, Department of Anesthesia, Critical Care and Pain Medicine, Brookline, MA; ${ }^{2}$ Division of Pain Medicine, Mount Sinai Medical Center, Miami, FL; ${ }^{3}$ Harvard Medical School, Boston, MA

Corresponding Author: Thomas Simopoulos, MD, Beth Israel Deaconess Medical Center, Department of Anesthesia, Critical Care and Pain Medicine, One Brookline Place, Suite 105, Brookline, MA 02445, E-mail: tsimopou@bidmc.harvard.edu

Conflict of Interest: None Declared

Disclaimer: There was no external funding in the preparation of this manuscript. Conflict of interest: Each author certifies that he or she, or a member of his or her immediate family, has no commercial association (i.e., consultancies, stock ownership, equity interest, patent/licensing arrangements, etc.) that might pose a conflict of interest in connection with the submitted manuscript. 


\section{BACKGROUND}

Interlaminar cervical epidural steroid injections (ICESI) are a relatively common procedure for the treatment of cervicalgia, cervical radicular pain, complex regional pain syndrome, and postlaminectomy cervical pain (15). Comprehensive review of the literature estimates that the complication rate of these procedures ranges between $0 \%$ and $16.8 \%(1,6)$. Complications associated with ICESIs may include headaches, vasovagal episodes, worsening neck pain, epidural hematoma or abscess, subdural block, intrathecal injection, dural puncture, spinal cord injury, and even death (6-8). A closed claims analysis of chronic pain procedures at the cervical spine revealed that $22 \%$ of all claims stemmed from cervical spine procedures and $31 \%$ of these resulted from direct needle trauma to the spine (2). Intramedullary cavitary lesions caused by needle insertion and delivery of injectate is exceedingly rare. Khan and Pioro (9) presented a report of cervical spinal cord syrinx formation as a result of cervical epidural injection which led to right hemiparesis and hemibody sensory loss below the neck.

Syringomyelia can cause dysesthesia, which is disabling and refractory to various available treatments, both conservative and invasive. Therapeutic modalities that have been described include neuropathic agents, nonsteroidal anti-inflammatory medications, opioids, regional sympathetic blocks, stellate ganglionectomy, and surgical collapse of the syrinx (10-11). Campos et al (12) successfully treated a patient with syringomyeliaassociated neuropathic pain refractory to medications with spinal cord stimulation (SCS). In the current case report we present a patient who developed a syrinx in the cervical spinal cord after needle puncture injury during an ICESI, with resulting neuropathic pain below the level of injury. Surgical implantation of a paddle lead SCS device yielded significant pain reduction, demonstrating the benefit of neuromodulation in mitigating neuropathic pain related to traumatic syringomyelia. A summary of the current literature reporting the use of cervical SCS for the treatment of nonradicular neuropathic pain syndromes of the upper extremity is reviewed.

\section{CASE REPORT}

The patient is a 52-year-old woman with a past medical history significant for irritable bowel syndrome, chronic bronchitis secondary to tobacco use, and chronic low back and neck pain. In her forties, her cervicalgia began to worsen with associated right arm pain, which led her to receive a series of cervical epidural steroid injections. Unfortunately, during an ICESI performed in 2010 at an outside hospital, the epidural needle was inserted into the cervical spinal cord and the injectate was administered directly into the cord. It was a right bias, C7-T1 interlaminar space injection under multiplanar fluoroscopy. The injectate consisted of one $\mathrm{mL}$ of preservative-free normal saline and $2 \mathrm{~mL}$ of methylprednisolone, $40 \mathrm{mg} / \mathrm{mL}$, and it was administered after the contrast medium flow was consistent with an epidural spread. This intraparenchymal injection resulted in transient bilateral lower extremity paralysis, permanent right upper extremity hand spasticity, and severe chest, lower back, upper and lower extremity pain, right worse than left. She was not sedated and distinctly recalled experiencing severe neuropathic pain, described as "lightning bolts," involving several anatomic locations which originated from the neck radiating to the arms and hands or from the groin extending cephalad towards the trunk or caudal towards the feet. She experienced neither urinary nor bowel incontinence.

Shortly after her ICESI and the appropriate work-up, she underwent surgery for cervical decompression with fusion, which did not alleviate her significant pain. After her surgery she received repeat ICESIs in an attempt to decrease her debilitating neck and bilateral radiating upper extremity pain. Despite physical therapy and trial of multiple antineuropathic agents, opioids, and muscle relaxants, her pain was unremitting. She also had low back surgery to address her low back and lower extremity pain, which was partially effective. Eventually, approximately 4 years after the event, she was referred to the neurosurgery department of our institution for possible further surgical intervention. Given that her magnetic resonance imaging (MRI) (Fig. 1) of the cervical spinal canal did not demonstrate evidence of any compressive lesion, it was determined that a trial of SCS would be a reasonable treatment option. She was then referred to our pain management center for an SCS trial.

During history intake, the patient's primary complaint was right upper extremity burning pain in a C7 dermatomal pattern. She also reported severe nondermatomal pain in many areas of her torso and her extremities, right greater than the left. Her musculoskeletal and neurological exam was notable for a right claw hand, 4 out of 5 strength in right thumb abduction, but otherwise 5 out of 5 strength in other upper and lower extremity myotomes. Her right biceps and triceps deep tendon reflexes were hyperreflexic, but her left upper and bilateral lower extremity reflexes were normal. Her Babinski reflex was negative bilaterally. Her sensation 
to light touch and pinprick were intact bilaterally in the upper and lower extremities. She had severe wasting of the thenar eminence on the right hand as well as severe spasticity of the intrinsic muscles (Fig. 2). The patient was on transdermal fentanyl at 75 mcg per hour every 72 hours, oxycodone sustained release $180 \mathrm{mg}$ per day in 3 divided doses, and oxycodone $30 \mathrm{mg}$ per day divided into 6 doses. Psychological evaluation revealed mild depression but no findings that would preclude a good outcome from SCS.

Under minimal sedation and using multiplanar fluoroscopy and the loss-of-resistance technique, a single 8-contact percutaneous lead (Abbott, Abbott Park, IL) was placed after the T2-3 interspace was accessed. The lead was advanced to the superior endplate of $\mathrm{C7}$ (Fig. 3A). The history of prior cervical decompression and fusion precluded further advancement. Once the patient reported satisfactory paresthesia coverage and perceived pain relief intraoperatively, even with suboptimal lead placement, the lead was secured with medical adhesives to the skin. During the trial, the patient experienced very good paresthesia coverage and significant pain reduction ( $50 \%$ pain intensity reduction) along with increased functional mobility of her upper extremity and improved quality of life. Four months after her SCS trial, she underwent permanent placement of a Penta (Abbott, Abbott Park, IL) paddle lead at the C7 level (Fig. 3b). The paddle type, though preferable if MRI-compatible, was selected based on its ability to give long-term coverage as well as surgeon preference and experience. At the 2-year follow-up following permanent implantation, she continued to report significant pain reduction of $40 \%$ in her right upper extremity, which was further aided with concur- rent use of pregabalin $600 \mathrm{mg}$ day, oxycodone $60 \mathrm{mg}$ per day in 3 divided doses, and $90 \mathrm{mg}$ of duloxetine. The patient was weaned off of all long-acting opioids. Her examination remained stable as depicted earlier in Fig. 2. Following the improvement in her right upper extremity, the patient for unclear reasons had diminished pain complaints in the other regions of her body. The patient

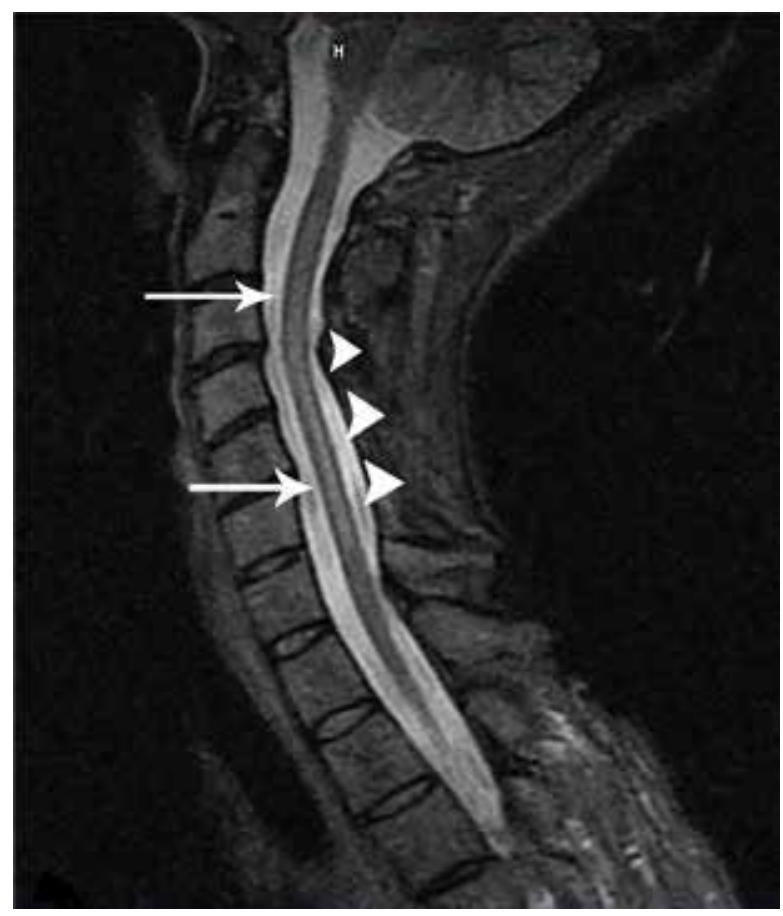

Fig. 1. Magnetic resonance imaging of cervical spine ShortTI Inversion Recovery sequence demonstrating central cervical cord hyperintensity associated with syringomyelia (arrows). Also demonstrated is a posterior laminectomy defect (arrow heads).
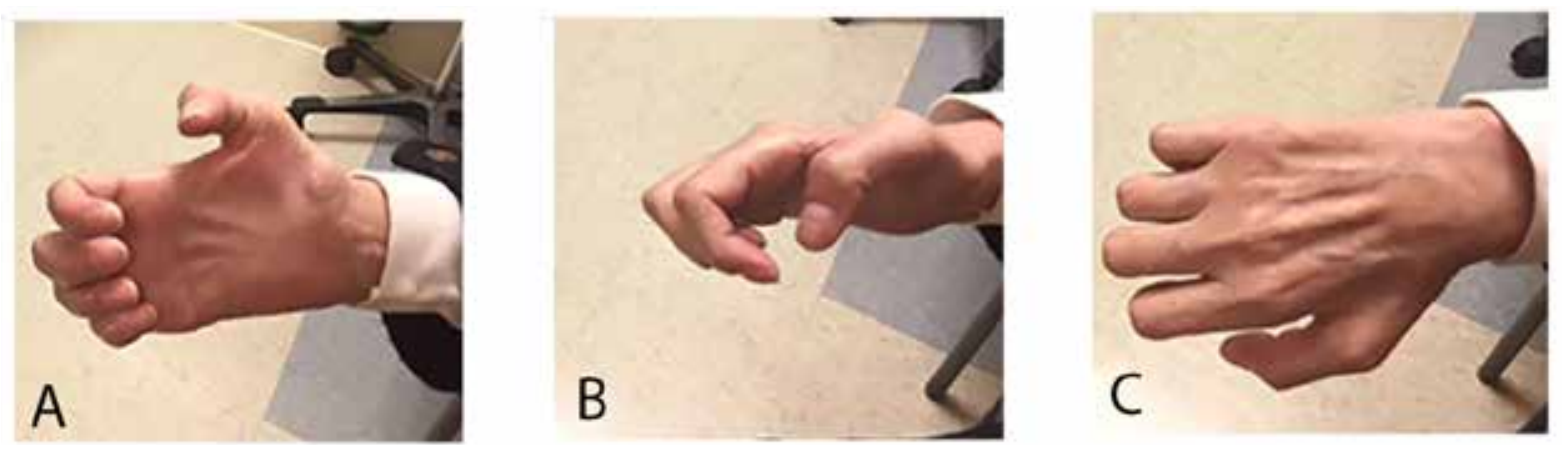

Fig. 2. Anterior (A), medial (B), and posterior (C) views of the right hand demonstrating intrinsic hand muscle spasticity and muscle wasting secondary to cervical cord lesion. 


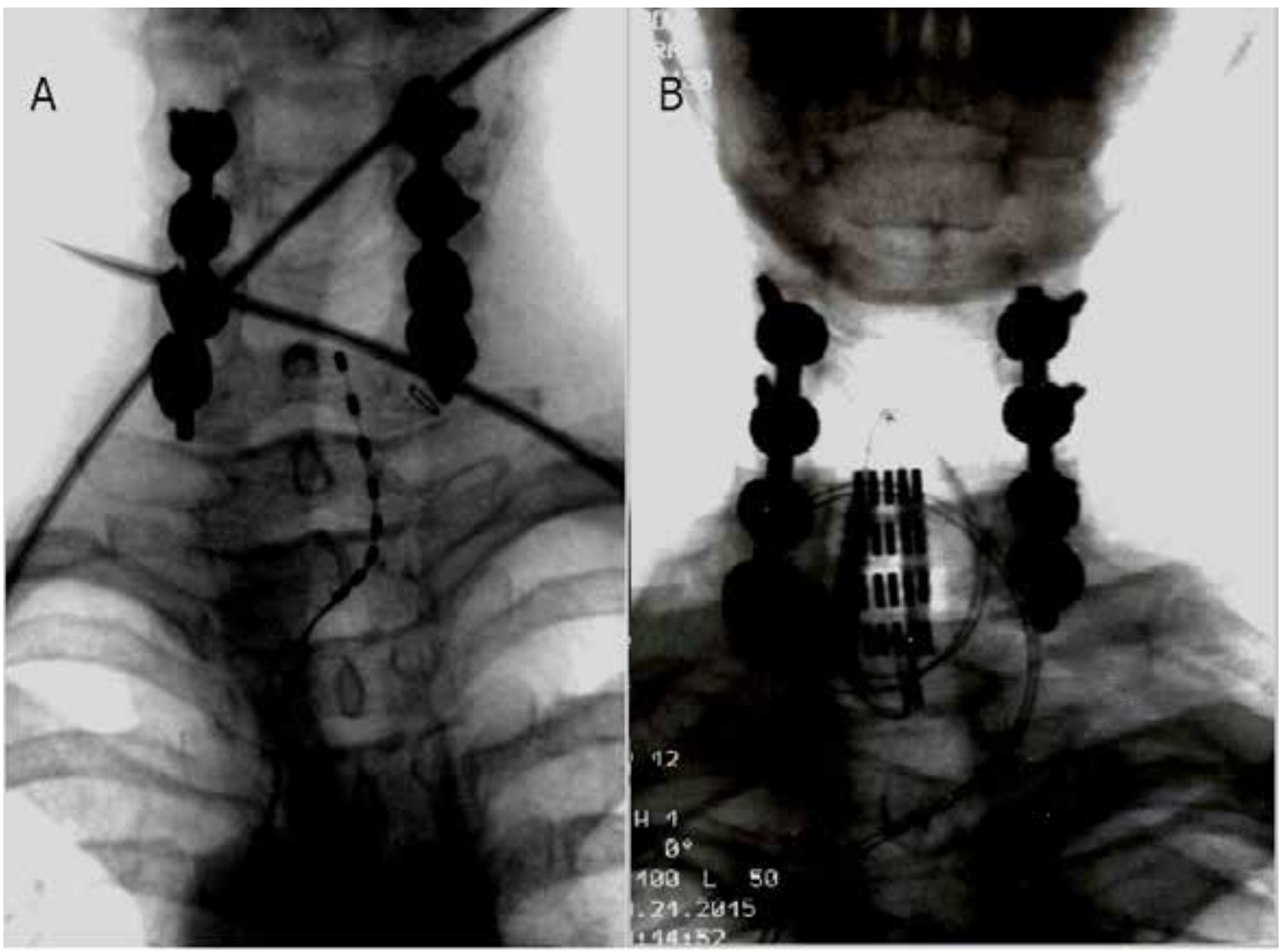

Fig. 3. (A) Fluoroscopic image of percutaneous trial of cervical spinal cord stimulator 8-contact Abbot lead. The superior lead contact at C7 is placed inferior to the laminectomy defect. (B) Abbot's PentaTM 5-Column Paddle Lead, cephalad contact at $C 7$.

felt paresthesias in her right upper extremity only and preferred this mode of stimulation over burst.

\section{DISCUSSION}

Cervical syrinx formation as a complication of ICESI is an exceedingly rare phenomenon with scant representation in the literature (9). The anatomy of the cervical spine is unique and different from that of the lumbar spine. Though the cervical epidural space at C7-T1 is the widest at this segment, the narrow distance between the ligamentum flavum and the spinal cord renders epidural injections in the cervical spine more precarious $(2,6,9,13,14)$. Multiplanar contrast medium-enhanced fluoroscopy used during ICESI may significantly mitigate the risk for complications (6).

Morelli et al (15) demonstrated that needle tract formation due to inadvertent spinal cord puncture can be associated with relatively little cord edema and manifest with significant, transient neurologic symptoms. In the case of syringomyelia, segmental dysesthesias associated with causalgia, hyperesthesia, and trophic changes may be observed (10). In patients with syringomyelia, it has been observed that the involvement of the dorsal column medial-lemniscus pathway increases the likelihood of experiencing neuropathic pain $(10,16)$. The dorsal horn and posterior column lesions in patients with syrinxes produce symptomatology patterns that are consistent with the hypothesis of deafferentation pain $(10,17-19)$.

In this case, the patient very quickly experienced myelopathic changes and significant neuropathic pain of central origin after an iatrogenic syrinx formation. Despite surgical decompression, physical therapy, and medication treatment, her pain remained rather debilitating. Milhorat et al (10), as well as Prat et al (20), demonstrated that in patients with surgical collapse 
of the syrinx, relief of dysesthesia occurred in $59 \%$ of patients, but $41 \%$ did not report improvement and in fact may have had worsening of their pain. Given the poor response to traditional treatment methods for controlling syrinx-related neuropathic pain, the use of SCS may be considered. Campos et al (12) successfully used SCS to treat neuropathic pain in a patient with atraumatic syringomyelia. They reported that the patient experienced significant reduction in the upper extremity neuropathic pain she was experiencing in addition to improved function and quality of life.

While there is a large body of evidence supporting the use of thoracic SCS in the treatment of lower extremity neuropathic pain, this is not the case for cervical SCS when one excludes cervical radiculopathy (Table 1). Most studies range from case reports to retrospective or prospective single center analyses. The unique anatomic characteristics of the cervical spine make a cervical SCS placement technically more difficult, carrying more inherent risks of neurological injury. These factors can lead to potential undertreatment of patients with upper extremity neuropathic pain.

Most case reports point to the feasibility that SCS may prove to be of high value in the treatment of refractory upper extremity neuropathic pain syndromes. For example, Chien et al (21) presented the case of a 42-year-old woman with a traumatic left upper extremity brachial plexus avulsion injury after a motor vehicle accident and treatment of deafferentation pain complicated by complex regional pain syndrome type II. Previous unsuccessful interventions included repeated stellate ganglion blocks, transcutaneous electrical nerve stimulation, and neuropathic and opioid medications. During the 7-day trial of a cervical SCS, the patient experienced immediate improvement of pain symptoms with the pain score decreasing from 9 out of 10 to 2 to 3 out of 10 on the Numeric Rating Scale (NRS-11). The left trial lead was placed at the C2-5 levels and the right lead at C4-7. Subsequently, a permanent dual cervical lead Octrode ${ }^{\circledast}$ SCS system connected to a 16-channel rechargeable implantable pulse generator (Saint Jude, Plano, TX) was permanently implanted. At the 10-month follow-up, her pain level remained stable at 2 out of 10 on the NRS-11 at rest and with activity, including aggressive physical therapy; her ability to perform daily activities had dramatically improved.

Another case of treating deafferentiation neuropathic pain after brachial plexus avulsion with the use of cervical SCS is reported by Abdel-Aziz et al (22). Their patient, a 25 -year-old man, reported a $50 \%$ pain reduction with the placement of a trial stimulator at the C3-6 levels and continued to have the same coverage and same reduction in pain levels after the surgical placement of the paddle stimulator at the C3-5 levels.

McLean et al (23) published a case report of a cervical SCS for the treatment of acute on chronic neuropathic pain occurring after dorsal decompression of $\mathrm{C} 5$ to $\mathrm{C} 7$ with instrumented stabilization from C4 to T2. More specifically, a 61-year-old man with a history of multiple cervical spinal cord surgeries complained of severe, diffuse, and progressive pain affecting the lower neck and arms bilaterally, left worse than right, on postoperative

Table 1. Summary of the current literature on cervical spinal cord stimulation for the treatment of nonradicular neuropathic pain.

\begin{tabular}{|l|l|l|l|}
\hline Author & Year & Type & Results/Endpoints \\
\hline McLean et al & 2018 & Case report & Cervical SCS treating acute on chronic postoperative upper extremity neuropathic pain. \\
\hline Levine et al & 2016 & $\begin{array}{l}\text { Retrospective single- } \\
\text { center study }\end{array}$ & $\begin{array}{l}\text { Cervical spinal cord and dorsal nerve root stimulation of 35 patients with neuropathic } \\
\text { upper limb pain. }\end{array}$ \\
\hline Abdel-Aziz et al & 2014 & Case report & $\begin{array}{l}\text { Treatment of deafferentiation neuropathic pain after brachial plexus avulsion with the use } \\
\text { of cervical SCS. }\end{array}$ \\
\hline Chien et al & 2014 & Case report & $\begin{array}{l}\text { Cervical SCS successfully treating deafferentation pain from brachial plexus avulsion } \\
\text { injury complicated by CRPS. }\end{array}$ \\
\hline Chivukula et al & 2014 & $\begin{array}{l}\text { Retrospective single- } \\
\text { center study }\end{array}$ & $\begin{array}{l}\text { Efficacy and outcomes of cervical and cervicomedullary spinal cord stimulation for } \\
\text { chronic pain in 121 patients. }\end{array}$ \\
\hline Campos et al & 2013 & Case report & SCS for the treatment of neuropathic pain secondary to syringomyelia. \\
\hline Kapural et al & 2008 & Case report & Cervical SCS for the treatment of lower extremity neuropathic pain. \\
\hline Forouzanfar et al & 2004 & $\begin{array}{l}\text { Prospective single- } \\
\text { center study }\end{array}$ & \begin{tabular}{l} 
Comparison of the efficacy of cervical and lumbar SCS in 36 patients with CRPS type I. \\
\hline
\end{tabular} \\
\hline
\end{tabular}

Abbreviations: CRPS, complex regional pain syndrome; SCS, spinal cord stimulation 
day one after the above-mentioned operation. The pain had a distribution pattern different from the previous neuropathic pain. His symptoms were so severe that they could only be controlled with ketamine infusion. The patient underwent a reexploration with a trial lead SCS placement. Intraoperatively, there was no evidence of medullary compression or hemorrhage. An A16-electrode SCS paddle lead (Specify 298, Medtronic Inc., Minneapolis, MN) was placed epidurally from C3 to C6. After a successful trial, a permanent implantable pulse generator was placed. At the follow-up visit, 12 months later, assessment of the patient's pain showed a pain intensity of 3 out of 10 on the NRS-11, compared to 9 out of 10 on postoperative day one.

An interesting case report by Kapural et al (24) describes a cervical SCS for the treatment of lower extremity neuropathic pain, refractory to multiple medications and epidural injections. Due to the patient's scoliosis and history of multiple lumbar spine surgeries, it was decided to place the leads in the lower cervical and upper thoracic regions to possibly capture the lower extremities. Two 8-contact leads with 4-mm spacing were guided through the patient's epidural space from the entry point at $\mathrm{T} 4$ to the $\mathrm{C} 5$ lead tip position, at which a test stimulation was performed. Although the patient complained of significant but tolerable upper extremity paresthesias, his lower extremity pain relief was so profound that he opted for the permanent implant with satisfactory results.

Forouzanfar et al (25), in a prospective study, compared the efficacy of cervical and lumbar SCS in 36 patients with complex regional pain syndrome (CRPS) type I. Using the 7-point Global Perceived Effect (GPE) scale, at least $42 \%$ of the cervical SCS patients and $47 \%$ of the lumbar SCS patients reported significant improvement. There were no differences between the cervical and lumbar groups regarding outcome measures.

Levine et al (26) reported their single-center experience with cervical spinal cord and dorsal nerve root stimulation (DNRS) for neuropathic upper limb pain. They proceeded with permanent implantation in 23 out of 35 patients based on a Visual Analog Scale (VAS) pain score decrease of more than $50 \%$ during trial stimulation. Both the SCS and DNRS groups had significant improvements in average VAS pain scores at 12 months compared with baseline, and the majority of patients in both groups obtained $\geq 50 \%$ pain relief. The pain relief was accompanied by opioid use reduction and improvement in the Short Form-36 quality-of-life scores.

The largest series of patients treated with cervical SCS, to our knowledge, is reported by Chivukula et al (27) as a single-center experience with cervical and cervicomedullary junction spinal cord stimulation in 121 patients. Of the 121 patients identified who underwent at least trial SCS, 100 underwent permanent lead implantation. Indications for cervical SCS were of broad etiology and most of them were cases of neuropathic pain, including: 8 cases of brachial plexus lesions, 33 cases of complex regional pain syndrome, 4 cases of degenerative disc disease, 23 cases of failed neck surgery syndrome, 6 cases of chronic radiculopathy, and 1 case of post-herpetic neuralgia. For the cervicomedullary junction SCS, indications included 10 cases of trigeminal deafferentiation pain, 4 cases of trigeminal neuropathic pain, 4 cases of PHN, and 7 cases of occipital neuralgia. The SCS were placed by one surgeon and the mean pain reduction averaged $56.6 \%$ at a mean follow-up of 4.2 years, showing very promising outcomes.

\section{CONCLUSION}

This case summary of cervical SCS for syringomyeliarelated neuropathic pain highlights a few cogent points. A traumatically induced case of syringomyelia can produce a centrally mediated refractory neuropathic pain state in addition to neurological deficits. SCS is a potential treatment option, although further studies are needed to elucidate its effectiveness. 


\section{REFERENCES}

1. Schreiber AL, McDonald BP, Kia F, Fried GW. Cervical epidural steroid injections and spinal cord injuries. Spine J 2016; 16:11631166.

2. Rathmell JP, Michna E, Fitzgibbon DR, et al. Injury and liability associated with cervical procedures for chronic pain. Anesthesiology 2011; 114:918-926.

3. House LM, Barrette K, Mattie R, McCormick ZL. Cervical epidural steroid injection: Techniques and evidence. Phys Med Rehabil Clin N Am 2018; 29:1-17.

4. Cohen SP, Hooten WM. Advances in the diagnosis and management of neck pain. BMJ 2017; 358:j3221.

5. Manchikanti L, Nampiaparampil DE, Candido KD, et al. Do cervical epidural injections provide long-term relief in neck and upper extremity pain? A systemic review. Pain Physician 2015; 18:39-60.

6. Abbasi A, Malhotra G, Malanga G, Elovic EP, Kahn S. Complications of interlaminar cervical epidural steroid injections: A review of the literature. Spine (Phila Pa 1976) 2007; 32:2144-2151.

7. Botwin KP, Castellanos R, Rao S, et al. Complications of fluoroscopically guided interlaminar cervical epidural injections. Arch Phys Med Rehabil 2003; 84:627-633.

8. Schreiber AL, McDonald BP, Kia F, Fried GW. Cervical epidural steroid injections and spinal cord injuries [published online ahead of print September 14, 2015]. Spine J 2016; 16:1163-1166.

9. Khan S, Pioro EP. Cervical epidural injection complicated by syrinx formation: A case report. Spine (Phila Pa 1976) 2010; 35:E614E616.

10. Milhorat TH, Kotzen RM, Mu HT, Capocelli AL Jr, Milhorat RH. Dysesthetic pain in patients with syringomyelia. Neurosurgery 1996; 38:940-946; discussion 946-947.

11. Masri R, Keller A. Chronic pain following spinal cord injury. Adv Exp Med Biol 2012; 760:74-88.

12. Campos WK, Almeida de Oliveira YS, Ciampi de Andrade D, Teixeira MJ, Fonoff ET. Spinal cord stimulation for the treatment of neuropathic pain related to syringomyelia. Pain Med 2013; 14:767768.

13. Bicket MC, Chakravarthy K, Chang D, Cohen SP. Epidural steroid injections: An updated review on recent trends in safety and complications. Pain Manag 2015; 5:129-146.

14. Van Boxem K, Rijsdijk M, Hans G, et al. Safe use of epidural corticosteroid injections: Recommendations of the WIP Benelux work group. Pain Pract 2019; 19:61-92.
15. Morelli JN, Porbandarwala N, Patel DV. Spinal cord needle tract following epidural steroid injection. J Vasc Interv Radiol 2012; 23:1543-1544.

16. Zeilig G, Enosh S, Rubin-Asher D, Lehr B, Defrin R. The nature and course of sensory changes following spinal cord injury: Predictive properties and implications on the mechanism of central pain. Brain 2012; 135:418-430.

17. Dotson RM. Causalgia-reflex sympathetic dystrophy-sympathetically maintained pain: Myth and reality. Muscle Nerve 1993; 16:1049-1055.

18. Simone DA, Baumann TK, Collins JG, LaMotte RH. Sensitization of cat dorsal horn neurons to innocuous mechanical stimulation after intradermal injection of capsaicin. Brain Res 1989; 486:185-189.

19. Roberts WJ. A hypothesis on the physiological basis for causalgia and related pains. Pain 1986; 24:297-311.

20. Prat R, Galeano I. Pain improvement in patients with syringomyelia and Chiari I malformation treated with suboccipital decompression and tonsillar coagulation. J Clin Neurosci 2009; 16:531-534.

21. Chien GCC, Candido KD, Saeed K, Knezevic N. Cervical spinal cord stimulation treatment of deafferentation pain from brachial plexus avulsion injury complicated by complex regional pain syndrome. A A Pract 2014; 3:29-34.

22. Abdel-Aziz S, Ghaleb A. Cervical spinal cord stimulation for the management of pain from brachial plexus avulsion. Pain Med 2014; 15:712-714.

23. Lawson McLean A, Kalff R, Reichart R. Spinal cord stimulation for acute pain following surgery for cervical myelopathy: A novel treatment strategy. Pain Pract 2019; 19:310-315.

24. Kapural L, Cywinski J, Zovkic P, Stanton-Hicks M. Effective cervical spinal cord stimulation for bilateral lower extremities pain: Technical report. Pain Pract 2008; 8:117-119.

25. Forouzanfar $T$, Kemler MA, Weber WE, Kessels AG, van Kleef M. Spinal cord stimulation in complex regional pain syndrome: Cervical and lumbar devices are comparably effective. $\mathrm{Br} J$ Anaesth 2004; 92:348-353.

26. Levine $A B$, Parrent $A G$, MacDougall KW. Cervical spinal cord and dorsal nerve root stimulation for neuropathic upper limb pain. Can J Neurol Sci 2017; 44:83-89.

27. Chivukula S, Tempel ZJ, Weiner GM, et al. Cervical and cervicomedullary spinal cord stimulation for chronic pain: Efficacy and outcomes. Clin Neurol Neurosurg 2014; 127:33-41. 
